\title{
Discussion on the relationship between power generation output of intermittent offshore energy and Marine Climate and Environment -- Taking wave energy and solar energy as examples
}

\author{
Lijie Zhao ${ }^{1 *}$ \\ ${ }^{1}$ School of Management, Tianjin University of Technology, Tianjin 300384, China
}

\begin{abstract}
In recent years, with the continuous promotion of China's marine power strategy, the comprehensive development and application of marine resources in many aspects and in depth has become a trend. China's South China Sea is vast and rich in resources, which has important national defense, economic and environmental significance for its comprehensive development and construction. The South China Sea is a vast sea area with abundant wave energy and solar energy. The pilot development and application of natural energy in this area is of great strategic and environmental significance for the decentralized power supply of deep-sea islands. Therefore, based on the actual application of the wave energy-solar power generation platform on YX Island, a comprehensive analysis is carried out based on the characteristics of the marine climate and environmental elements of the South China Sea, exploring the apparent efficiency of intermittent energy such as wave energy and solar energy under specific environmental conditions and putting forward relevant suggestions. The forecast will provide relevant theoretical basis and data support for the subsequent in-depth development, application and system optimization of renewable energy in the South my country Sea.
\end{abstract}

\section{Introduction}

As the gateway to the South China Sea, SS city is in an extremely important strategic position. As the political, economic and military center of SS city, YX Island is a typical deep-sea island in China. Without power supply from the mainland grid, it mainly relies on ships to transport diesel and fuel oil for power generation, which has problems such as high cost of power generation and limited resources affected by fuel inventory. The comprehensive and efficient development of resources related to YX Island and its associated islands is hindered by the shortage of conventional energy. China's South China Sea has a vast sea surface and a long coastline, with good wave energy. ${ }^{[1]}$ It is of great significance for the power supply of distributed deep-sea islands to develop wave energy locally and realize wave energy nearby and stable power generation. At the same time, the South China Sea has vast sea surface and excellent photovoltaic and wind energy resources. If wave energy, photovoltaic and wind energy can be combined to form a stable multienergy complementary model, the stability and overall efficiency of the renewable energy system will be effectively improved. In recent years, with the support of the state, the wave power generation technology in China has made a key breakthrough, and the large semisubmersible wave power generation device technology has been piloted and connected to the grid in relevant islands. This technology provides an opportunity to install renewable energy capture systems such as solar and wind power on wave power generation devices.

At present, there have been some cases in China to develop island energy comprehensive application by using large semi-submersible wave power generation platform technology. ${ }^{[2]}$ On this basis, adding intermittent energy capture and conversion modules such as solar energy to form a multi-energy complementary system power supply will face new difficulties. Among them, the influence of local ocean temperature and humidity on the apparent performance of intermittent energy modules such as wave energy and solar energy is an important aspect. China's South China Sea has special sea conditions, such as perennial high temperature, high humidity, typhoons and other climatic conditions, which have a great impact on the operational stability of wave energy, solar energy and other renewable energy capture and conversion system. ${ }^{[3]}$ Full investigation and analysis of wave energy, solar power and other intermittent energy output module under the condition of the local Marine climate environment performance and stability of the situation, through the analysis, carry out scientific prediction and application demonstration, after comprehensive comparison for the follow-up of the south China sea contains extensive wave energy, solar power and other intermittent renewable energy power generation and comprehensive application to provide detailed and scientific theory basis.

\footnotetext{
*Corresponding author: 398108024@qq.com
} 


\section{Overview of climatic and environmental elements in the waters of YX Island}

At present, the large semi-submersible wave energy comprehensive application platform developed by Guangzhou Institute of Energy, Chinese Academy of Sciences has carried out the wave energy power grid connection test in the waters off YX Island in the South China Sea. Therefore, the following data collection and analysis will mainly start from the factors such as solar radiation, water vapor, salinity, temperature and humidity in the waters around YX Island.

\subsection{Solar radiation and temperature conditions}

YX Island has high solar radiation and high temperature all year round, with an average temperature of $26.8^{\circ} \mathrm{C}$ for many years. Under the regulation of the ocean, the annual temperature difference is not big, the amplitude is around $6{ }^{\circ} \mathrm{C}$, the extreme maximum temperature is $35^{\circ} \mathrm{C}$, the extreme minimum temperature is $16.4^{\circ} \mathrm{C}$.

\section{2 moisture}

YX island has sufficient water vapor sources and abundant precipitation resources, but the seasonal distribution is relatively large, with annual precipitation of $1382 \mathrm{~mm}$. The maximum precipitation occurred in September, reaching $302.3 \mathrm{~mm}$. At least in February, only $12.8 \mathrm{~mm}$. The annual precipitation days of YX Island are 117 days, and the annual average precipitation intensity is $11.8 \mathrm{~mm} / \mathrm{d}$.

\section{3 salinity}

The salinity of the sea area around YX Island is generally high, above 33.4. In February, the precipitation was the lowest, so the salinity was the highest (34.0). From September to October, there was more precipitation and slightly lower salinity, which was 33.4.

\subsection{Overview of temperature and Humidity}

The experimental sea area is located in the south of the Tropic of Capricorn.it has a tropical monsoon climate with an annual precipitation of $1509.8 \mathrm{~mm}$. Because be surrounded by sea and land area is small, have the characteristic of maritime climate, rainfall is abundant, year round high temperature, high humidity, high salt, air is clean, sunshine is bright, ultraviolet ray is particularly intense. The annual average temperature is $26.5^{\circ} \mathrm{C}$ with more direct sunlight and longer sunshine. January is the coldest month with an average temperature of $23^{\circ} \mathrm{C}$. June is the hottest month with an average temperature of $29^{\circ} \mathrm{C}$. The maximum daily temperature is $31{ }^{\circ} \mathrm{C}$ and the minimum is $21^{\circ} \mathrm{C}$. The rainy season is from May to June every year. The seasonal variation of ocean surface water temperature is consistent with the year-round distribution of water temperature.

\subsection{Potential application impact}

The climatic and environmental conditions radiated from the waters of YX Island to the whole Waters of the South China Sea and their periodicity have certain similarities. From the perspective of photovoltaic resources, the application of solar energy capture and conversion in this sea area does not need to consider the problem of resource conditions. However, the large salinity and humidity in this area have a great corrosion effect on wave energy conversion system equipment and photovoltaic solar panels. When setting up the intermittent energy system, factors such as water vapor invasion and corrosion prevention should be fully considered; Before the wave energy application test is carried out, the overall wave condition of the surrounding sea area is generally investigated and the appropriate release point is selected. The wave conditions, terrain and other factors involved in this part are not the focus of this paper. However, the south China Sea is open, so it can be considered that the radiation is uniform at any location, so it is unnecessary to give priority to the selection of wave energy equipment.

\section{A Brief Analysis of wave energy and Solar Power Efficiency}

The sea conditions in the South China Sea are complex and the climate is bad. From May to November every year, it is the season with dense typhoons. The wave energy and solar power efficiency in July and August 2018 are selected for analysis below.

\subsection{Wave generation data}

\subsubsection{Generation time}

On July 14, 2018, the commissioning of the wave-power generation device was completed and the test power generation began. By July 26, the relevant departments of the local sea area carried out the mission and suspended the test until August 6. During the normal test in August, the power generation module of wave energy was locked from August 18th to August 26th, and the power generation function of wave energy was gradually unlocked after August 26th due to the influence of typhoon.

\subsubsection{Generation output of wave energy module}

According to the power generation data and operation status records of the device, the wave power generation device in the designated sea area of YX Island has a good wave response, mainly because the open sea leads to more sporadic waves, which intuitively reduces some unnecessary negative feedback in the inland sea. The apparent efficiency of wave power generation module is better than that in the inner sea, mainly due to the open sea, neat wave condition and long wave length in the South China Sea. Therefore, it can be considered that the higher temperature and humidity in this region have little influence on the wave power generation and output 
module.

\subsection{Solar power data}

Solar power generation data for July and August 2018 are shown in Figures 1 and 2, respectively.

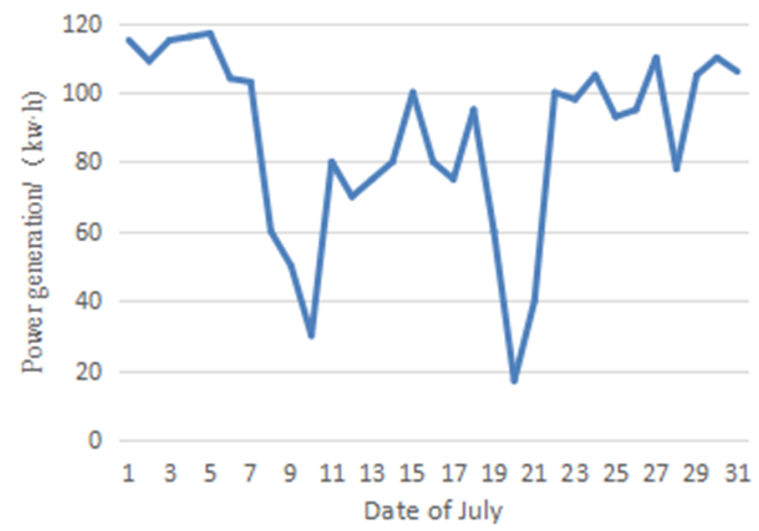

Fig. 1. Solar power generation data curve for July 2018

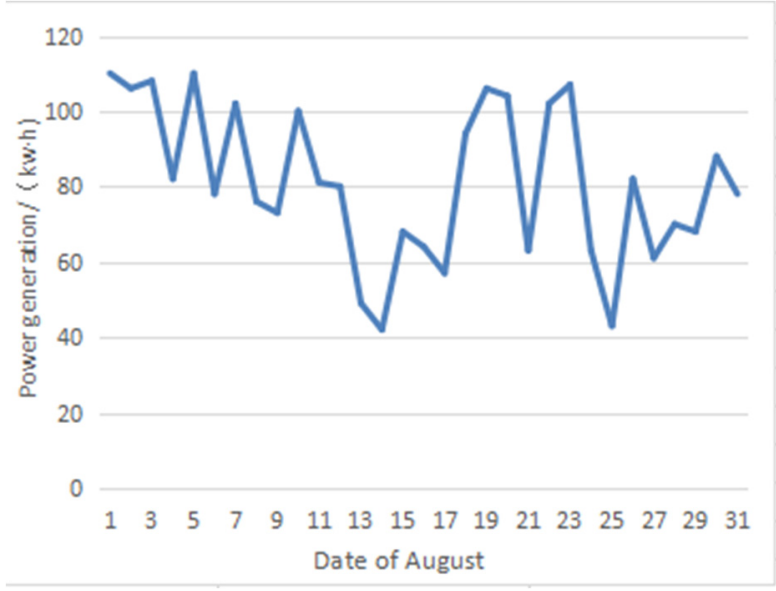

Fig. 2. Solar power generation data curve for August 2018

\subsubsection{Generation time}

The operation of the solar energy module is not affected by the locked wave power generation module when the wind and waves are high. The solar energy module can generate power sustainably, and the power generation time is from July to August 2018.

\subsubsection{Solar power output}

The installed capacity of the solar energy module of this test platform is $60 \mathrm{~kW}$, and the average daily generating capacity is within the reasonable normal range of $80 \sim$ $100 \mathrm{~kW} \cdot \mathrm{h}$. Under the influence of cloudy days caused by typhoons or thunderstorms, photovoltaic power generation efficiency is below the average level for 5-8 days per month, which is within the normal range.

\subsection{Brief analysis of intermittent energy generation output}

According to the apparent data, the wave power generation module performs normally in the test area. The water depth in the test sea area is relatively large, but based on the special anchoring and fixed mode of the wave power generation platform, the stability of the platform is basically not affected, which is conducive to the normal and stable operation of the wave power generation module. The wave conditions in the open sea where the test site is located are better than those in the inner sea, and the power output performance of the wave power generation module on the same platform is better than that in the Inner Sea (Wanshan Sea area, Zhuhai), with better rated power generation time and power generation efficiency. After inspection, the relevant performance equipment of wave power generation module is normal under the condition of water vapor prevention and corrosion prevention. It can be preliminarily considered that the higher temperature, humidity and salinity in the South China Sea have little influence on the output of wave power generation. In terms of solar power generation output, mainstream solar photovoltaic panels are generally used at present and have waterproof functions. From July to August, the sea water vapor has little influence on the PHOTOVOLTAIC plate during the period of strong light in the South China Sea. The salt factor belongs to the adhesion and accumulation type influence, which does not accumulate to the level that affects the photovoltaic power generation efficiency temporarily during the test. At the same time, according to the application specifications of the wave energy integrated power generation application platform, the offshore equipment and instruments will be irregularly repaired during the test period, including the cleaning and inspection of all the equipment of the solar power generation output module, so as to minimize the influence of salt or other impurities on the solar power generation output.

\section{The intermittent type energy generation output and the South China Sea climate environment relations}

The key to the comprehensive application and practice of intermittent renewable energy power generation platforms including wave energy and solar energy in the South China Sea is the stability and reliability of the platform operation, and the core is the power generation efficiency and sustainability of intermittent wave energy and solar energy.

\subsection{Environmental coordination should be emphasized in the application of island intermittent renewable energy power generation output}

On the basis of the structure of large semi-submersible waves can be combined with solar, wind and other renewable energy power generation is a new technology platform, in addition to carry out the application before taking topographic survey, wave, solar and wind energy measurement, should pay attention to the platform on the sea area the ecological effects of the target, may not cause damage to the local original ecological or weakened. This is the basic premise for the development and application 
of intermittent multi-energy complementary energy systems in the South China Sea.

\subsection{Influence of high temperature, high humidity and high salt in the South China Sea on the power generation output of intermittent energy platform}

Based on the test of the existing wave-solar power generation platform on YX Island, it is found that the high temperature, high humidity and high salt environment in the South China Sea has little impact on the power generation output of the intermittent energy platforms containing wave energy and solar energy. On the one hand, the offshore operation mode of the wave power generation module in the sea area in this region has been relatively mature, relevant measures to deal with various sea conditions have been relatively complete, and climatic conditions such as temperature and humidity have little influence on its main performance. On the other hand, photovoltaic solar technology has been developed for many years, and the application mode on land has been very mature. On the basis of solid protection at sea, it can be transplanted to the floating working platform on the sea, and its inherent characteristics can also be given full play. Due to the low latitude and high perennial temperature in the South China Sea, there is no problem of low temperature affecting the activity of the mechanism or equipment. At the same time, July-August is the period with high humidity in the South China Sea, and the other periods with low humidity are more conducive to the operation and maintenance of the equipment. Therefore, the preliminary prediction of temperature, humidity and other environmental factors on the intermittent energy generation output has little impact. However, attention should be paid to the problem of salinity corrosion in the area. The internal system should be strengthened sealing and inspection, while the external system should be cleaned and maintained regularly. A timed automatic cleaning device can be set to carry out intermittent cleaning on the surface of the integrated energy platform.

\subsection{Comprehensive consideration of advantageous energy of islands}

In China, the islands in the South China Sea are numerous and scattered, and the construction of fixed power stations on the islands is also faced with the challenge of the climatic and environmental conditions in the sea area. For islands with superior energy forms and stocks, such as wave energy and solar energy, full consideration can be given to converting wave energy and solar energy intermittent energy into conventional energy. Its overall construction and maintenance costs will be lower than building a conventional fixed power station, and it will be more environmentally friendly.

\section{Conclusion}

On the premise of meeting the relevant environmental applicability and special regional management requirements, and with the climate and environmental characteristics such as temperature and humidity in the South China Sea, ${ }^{[4]}$ it is more suitable for the comprehensive application of intermittent energy power generation output including wave energy and solar energy, and can further develop the development and application of other renewable energy such as wind energy. ${ }^{[5]}$ Compared with the inland sea, in the south China Sea, in addition to the vast sea surface, measures should be taken to cope with large waves, and further anti-corrosion and water-vapor intrusion work should be done. On this basis, the more abundant energy resources in the open sea of the South China Sea will be conducive to the operation of the energy capture and conversion system. Note that in the south China sea were extensively developed, including wave energy, solar energy, wind energy, renewable energy application is a large area, about the sustainable development of the national and local strategic deployment work, need a multisectoral, multi-disciplinary, multi-industry comprehensive participation, is a long-term work over and over again, need to advance step by step, finally to deep sea of renewable energy in our country comprehensive positive demonstration, system development and application of high quality to promote the implementation of the strategy of national Marine power development.

\section{References}

1. Pan X, Huang Y, Tang J, Yang L. Analysis of influencing factors and prospect of new energy power generation development[J]. Smart Power.,47(11):4147(2019).

2. Wang Q. Analysis and Prospect of offshore wind power development in the southeast Coast of China[J]. Jiangxi Fishery Science and Technology. 5,4344(2018).

3. Chen J. Development trend and enlightenment of Offshore wind power in the United States. Reformation \& Strategy. 30(10):129-134(2014).

4. Zhou D. Offshore wind power becomes a new "wind outlet" for energy industry investment. Sino-Global Energy.23(01):54(2018). 